\title{
Enantioselective Protonation
}

\author{
Justin T. Mohr, Allen Y. Hong, and Brian M. Stoltz \\ Arnold and Mabel Beckman Laboratories of Chemical Synthesis, Department of Chemistry and \\ Chemical Engineering, California Institute of Technology, 1200 East California Boulevard, Mail \\ Code 164-30, Pasadena, CA 91125 (USA)
}

\section{Abstract}

Enantioselective protonation is a common process in biosynthetic sequences. The decarboxylase and esterase enzymes that effect this valuable transformation are able to control both the steric environment around the proton acceptor (typically an enolate) and the proton donor (typically a thiol). Recently, several chemical methods to achieve enantioselective protonation have been developed by exploiting various means of enantiocontrol in different mechanisms. These laboratory transformations have proven useful for the preparation of a number of valuable organic compounds.

A fundamental method to generate a tertiary carbon stereocenter is to deliver a proton to a carbanion intermediate. However, enantioselective transfer of a proton presents unusual challenges, specifically, manipulating a very small atom and avoiding product racemization at a particularly labile stereocenter. As a result, the conditions for a successful enantioselective protonation protocol may be very specific to a certain substrate class. Tertiary carbon stereocenters are extremely common in valuable biologically active natural products, and thus the need for synthetically useful enantioselective methods to form these stereocenters is vital. 1

In this review, we discuss several strategic approaches to enantioselective protonation. Emphasis has been placed on recently developed methods and their accompanying mechanisms in order to update the most recent prior reviews on this topic..$^{2,3,4,5,6,7,8}$ Each method relies on particular stereochemical control elements based on the mechanism of the protonation transformation. Appreciation of these controlling elements may lead to improved methods for preparing valuable chiral materials for a variety of synthetic applications.

\section{Important Factors in Achieving Enantioselective Protonation}

Several of the most important practical features of enantioselective protonation were enumerated in Fehr's 1996 review. ${ }^{2}$ Principal among these is the fact that enantioselective protonations are necessarily kinetic processes since under thermodynamic control racemate would be formed. Accordingly, it is often necessary to match the $\mathrm{p} K_{\mathrm{a}}$ of the proton donor and the product to prevent racemization before product isolation. It is unfortunate that the same anion stabilizing groups (e.g., ketones) that make protonations relatively easy to achieve also impart a degree of instability in the product. This has led some researchers to explore hydrogen atom transfer reactions in lieu of Brønsted acid-mediated protonations (see the subsection Enantioselective Hydrogen Atom Transfer below).

Correspondence should be addressed to B.M.S. stoltz@ caltech.edu.

The authors declare no competing financial interests. 
In addition to the obvious challenges of product stability under the reaction conditions, the rapid rate of proton exchange in solution often leads to significant levels of background reaction without the intercession of the chiral control element. As a result, typical proton donors are relatively weak acids that react with the proton acceptor in a slower and more controlled fashion.

Since substrates for enantioselective protonation generally involve a prochiral $\mathrm{sp}^{2}$-hybridized atom, the stereochemistry of the substrate is a concern. In some cases, the ability to generate stereodefined proton acceptors (e.g., a pure $E$ - or $Z$-enolate) is critical to the success of a protonation method. In other cases, however, the two stereoisomers of enolate may in fact lead to the same enantiomer of product. ${ }^{5}$ To obviate this concern many researchers choose to investigate cyclic substrates; in turn, this may lead to a limited substrate scope for a particular system. The method to access the reactive proton acceptor is among the most important facets of each protonation system, and many strategies have been explored (e.g., conjugate addition, addition to ketenes, and decarboxylation from $\beta$-ketoesters).

Finally, the fine mechanistic details of enantioselective protonations are often not well understood. Since typical proton acceptors are stabilized anions, there are multiple Lewis basic sites available for protonation. It is likely that these sites protonate at kinetically different rates dependent on the specific reaction conditions. Potentially, enantioselective protonations may be achieved either by direct protonation to generate the desired stereocenter, or by protonation at a different site followed by enantioselective tautomerization. In an important recent report, Fehr ${ }^{9}$ demonstrated that isolated enol 1 (Figure 1) could be transformed enantioselectively into ketone 4 (an immediate precursor to the rose-smelling fragrance compound $(S)$-( $\alpha$ )-damascone) via the proposed aggregate complex $\mathbf{3}$, and this mechanistic course seemed to be operative in the analagous protonation of a lithium enolate with the conjugate acid of alkoxide $\mathbf{2}$. Based on these findings, perhaps some protonation protocols are more accurately described as enantioselective tautomerization reactions. Although ultimately inconsequential in terms of the products obtained, insights into the specific mechanistic course of the reaction are important in order to improve these systems. However, only rarely have these levels of mechanistic understanding been realized.

\section{Enantioselective Protonation in Enzymatic Systems}

Nature has evolved several efficient enzymes that catalyze enantioselective protonation reactions on useful organic building blocks. In recent reports, decarboxylases and esterases have proven to be two popular classes of natural enzymes for the construction of $\alpha$-stereocenters adjacent to ketones. Esterases release latent enolates from prochiral substrates while decarboxylases generate enolates in situ from malonic acid derivatives (Figure 2).

Ohta and coworkers10,11 isolated arylmalonate decarboxylase (AMDase) from Gram-negative bacterium Alcaligenes bronchisepticus and found that it catalyzes the decarboxylative enantioselective protonation of $\alpha$-aryl- $\alpha$-methyl-malonates through the proposed mechanism in Figure 2a. Yields and enantiomeric excesses were excellent for substrates with various $\alpha$ aryl substitutents (up to 99\% yield and 99\% ee). Experiments have shown that the Cys 188 residue is essential for activity, and this site is the putative proton donor that stabilizes the enolate intermediate. A Hammett study ${ }^{12}$ of the reaction found a $\rho$ value of +1.19 , which is consistent with a negatively charged transition state. 10 Recently, preliminary X-ray diffraction experiments and an X-ray crystal structure of AMDase were reported.13,14

Accessing the opposite enantiomeric series of products required additional investigation. ${ }^{15}$,

16 Analysis of the enzyme amino acid sequence was carried our to check for homology with known enzymes. The Cys 188 residue is conserved in several racemase enzymes from other microorganisms. Glutamate racemase, found in the bacterium Lactobacillus fermenti, contains 
an active site similar to that of AMDase, but with cysteine residues (Cys188 and Cys74) on both sides of the substrate. Presumably, one of the cysteine residues acts as a base and generates an enolate intermediate which can then be protonated non-selectively from either cysteine residue to give rise to a racemic mixture. When Ohta and coworkers prepared a G74C mutant of AMDase to mimic these racemase enzymes, they found that racemic $\alpha$-thienylpropionic acid (6, Figure $2 \mathrm{a}$ ) was formed in $37 \%$ yield from the malonic acid substrate (5). Further explorations based on this homology hypothesis led to the preparation of a double mutant of AMDase

$(\mathrm{G} 74 \mathrm{C} / \mathrm{C} 188 \mathrm{~S})$ that removed the native cysteine residue while maintaining the mutant residue on the opposite face of the substrate. The opposite enantiomer of product was indeed obtained with this new enzyme in $94 \%$ enantiomeric excess, although yields decreased to $60 \%$ and the activity of this mutant was several orders of magnitude lower than the wild-type. Some activity was rescued by performing random mutagenesis and identifying more active triple mutants. ${ }^{17}$

Decarboxylase-type activity was also observed in the conversion of $\beta$-hydroxyacid 7 to optically active $\alpha$-arylpropionic acid $(R)-\mathbf{1 1}$ by Gram-positive bacteria Rhodococcus $s p$. KU1314 (Figure 2b). ${ }^{18}$ In the proposed metabolic pathway, enzymes in the microorganism non-selectively oxidize hydroxyacid substrate $\mathbf{7}$ to aldehyde $\mathbf{8}$ and then decarboxylate the corresponding acid to form enolate $\mathbf{9}$ that undergoes enantioselective protonation to generate aldehyde 10. Subsequent non-selective oxidation affords enantioenriched $\alpha$-arylpropionic acid 11. Mechanistic experiments were consistent with this proposed enantioselective protonation mechanism rather than alternative possibilities such as enantioselective oxidation steps. Enantioselectivity and yield varied considerably depending on the aryl and alkyl groups at the $\alpha$-position in the substrate, but enantiomeric excesses up to $85 \%$ could be achieved. Employing an electron poor aryl group appeared to give poor enantioselectivity due to increased acidity of the proton in the presumed aldehyde intermediate. When the alkyl group was changed from methyl to ethyl, the reaction yield dropped, suggesting that the enzyme is sensitive to sterics.

Several researchers have employed esterases to obtain enantioenriched protonation products. In 1990, Ohta and coworkers ${ }^{19}$ reported that live Pichia miso IAM 4682 yeast cells catalyze the conversion of enol acetates to enantioenriched ketones (e.g., $12 \rightarrow \mathbf{1 3}$, Figure 2c). High levels of enantiomeric excess were attained with a variety of enol esters. For larger ring systems, the reaction yield and absolute configuration varied unpredictably with ring size. In an impressive application, this yeast-mediated reaction was used to generate an $\alpha$-stereocenter in a 12-membered ring with $96 \%$ ee.

Hirata and coworkers ${ }^{20}$ reported that liverwort Marchantia polymorpha esterase I also catalyzes the same reaction on a variety of substrates with differing alkyl side chains, but the facial preference for proton delivery varied for different enolate substitutions. For example, the enzyme delivered $(S)$-2-methylcyclohexanone $(\mathbf{1 3})$ and $(R)$-2- $n$-propylcyclohexanone from their respective enol acetates in $99 \%$ conversion and $99 \%$ e.e.

Lipase PS-C II, originating from Gram-negative bacteria Burkholderia cepacia, may also be used for the hydrolysis of 1-acetoxy-2-methylcyclohexene (12). Sakai and coworkers ${ }^{21}$ discovered that the enantiomeric excess of the product $((R)-13)$ was largely dependent on the temperature and the proton source. The best results were obtained by running the reaction at $0{ }^{\circ} \mathrm{C}$ with solid-supported enzyme PS-C II and ethanol as proton source (82\% conversion, $77 \%$ e.e.).

Among these enzymatic approaches, a general problem appears to be the difficulty of enzyme modification to give the unnatural antipode of product. Another limitation is the need for buffers to help stabilize enzymes or cells. Substrate scope is also limited due to the specificity of substrate recognition. For these reasons, enzymatic reactions do not provide a general solution to the synthesis of enantioenriched protonation products. Laboratory means for 
enantioselective protonation may enable a more universal protocol due to the ability to tune the structural and electronic features of the catalyst. These natural systems do, however, demonstrate many of the key controlling elements necessary for successful enantioselective protonation.

\section{Strategic Approaches to Nonenzymatic Enantioselective Protonation}

Using enzymatic systems as a guide to the important factors in achieving enantioselective protonation, two distinct factors have been envisioned as opportunities for asymmetric induction: the use of a chiral Brønsted acid (see the subsection Enantioselective Protonation by Means of Chiral Proton Donor below) and generation of a chiral proton acceptor intermediate (see the subsection Enantioselective Protonation by Means of Chiral Brønsted Base below). Whereas the enzymatic systems exploit both of these control elements, many laboratory methods have sought to use only a single control element not only to minimize the amount of enantiopure material required for the transformation, but also to eliminate complicating diastereomeric interactions possible in systems with multiple chiral additives. In practice, some of these systems seem to involve protonation through an aggregate complex of both proton acceptor and proton donor, typically in a metal complex (for example, Fehr's tautomerization depicted in Figure 1).

An additional factor important in improving efficiency of enantioselective protonation systems is achieving catalysis. For example, catalytic generation of a chiral metal/enolate complex in situ minimizes the amount of chiral controller required. Alternatively, coupling of a catalytic chiral proton donor to a stoichiometric achiral proton source achieves a similarly efficient use of chiral information. In the latter case, however, a specific order of thermodynamic acidity of the reaction components must be employed (necessarily in this order of decreasing Brønsted acidity: stoichiometric proton source, catalytic chiral protonating agent, and product). An accompanying balance of kinetic rates of proton transfer between all of these components must also be achieved to allow a reasonable rate of protonation through the catalyzed pathway while avoiding undesired background reaction between the prochiral proton acceptor and the stoichiometric achiral proton donor. Vedejs and coworkers have disclosed a study of these factors that is representative of these important issues. ${ }^{22}$

\section{Enantioselective Protonation by Means of Chiral Proton Donor}

Perhaps the most fundamental means of achieving an enantioselective protonation is to employ a chiral proton donor. The acidic proton often comes from an oxygen, nitrogen, or carbon atom in the proton donor. Indeed, the earliest enantioselective protonation protocols employed this technique.

Among the most popular substrates for enantioselective protonation are lithium enolates, which are often generated from ketones, enol acetates, or silyl enol ethers at low temperatures. This method most closely resembles an esterase approach taken by Nature. Kim and coworkers ${ }^{23}$ have synthesized a family of hydroxyethers chiral proton sources (e.g., 16, Figure 3a) capable of protonating lithium enolates of tetralones and indanones (prepared in situ from silyl enol ethers such as 14) in up to $97 \%$ yield and $90 \%$ e.e. The acidity of the Brønsted acids had a strong correlation to enantioselectivity and salt-free conditions were important to selectivity. A $\pi-\pi$-stacking interaction between the substrate and rigid proton source was proposed as the chiral controlling interaction during the protonation event. Since cyclohexanone-derived enolates lack an aryl group to participate in the stacking interaction, poor e.e. was observed for these substrates.

Eames and coworkers $24,25,26$ have developed several strategies for the asymmetric protonation of prochiral tetralone enolates based on structurally different chiral proton sources. 
In one example, tris(sulfonamide) protonating agents with chiral backbones (e.g., 18, Figure $3 b$ ) provided yields of ketone products (e.g., 15) up to $70 \%$ and enantiomeric excesses of up to $77 \% .{ }^{26}$ In these systems, it is believed that the enolates and proton sources can form an organized transition state that is guided by lithium chelation and perhaps more closely resembles a chiral aggregate intermediate than a direct enolate protonation. In some cases it was possible to access the opposite antipode of the product ketone by employing an external quench strategy. ${ }^{24}$

Enolate intermediates can also be accessed through decarboxylation. Proton donors derived from Cinchona alkaloids (e.g., 21, Figure 3c) have proven to be especially useful reagents for this enantioselective protonation strategy. Rouden and coworkers ${ }^{27,28}$ have shown that cyclic and acyclic $\alpha$-aminomalonate hemiester substrates (e.g., 19) can be protonated with high levels of enantioselectivity. Enantiomeric excesses of product esters (e.g., 20) up to $93 \%$ could be achieved in cyclic cases and 89\% in acyclic cases. Products in the opposite enantiomeric series could be generated in comparable e.e. using catalysts prepared from naturally occurring diastereomeric alkaloids. The alkaloid derivatives are believed to serve as dual-purpose reagents: they deprotonate malonate hemiester substrates and promote a decarboxylation event. The intermediate enolate can be protonated by the tertiary ammonium salt to give enantioenriched products. This approach is biomimetic and resembles decarboxylase enzymes in Nature.

Work done by Donohoe and coworkers ${ }^{29,30}$ has shown that it is possible to perform dissolving metal reductions of pyrrole esters and quench the resulting enolate intermediates with a chiral proton source. Reduction of 2,5-disubstituted pyrroles (e.g., 22, Figure 3d) led to a separable 1:1 mixture of trans- and meso-diasastereomers of ester $\mathbf{2 4}$, but the asymmetric induction in the chiral trans-product was good when (-)-ephedrine (23) was used as the chiral proton source. Based on enantioselectivities of reactions with substituted ephedrine derivatives, it was proposed that the hydroxyl group provides the proton and that the ephedrine molecule needs to interact with the lithium cation in a bidentate fashion for optimal asymmetric induction.

Considering this proposal, this transformation may be more accurately described as protonation through a chiral aggregate of Brønsted acid and base. A related transformation of pyrrole monoesters with oxazolidinone proton donors yielded reduced products in up to $68 \%$ ee and $58 \%$ yield. These partially reduced pyrroles could be elaborated to form uncommon dihydroxylated amino acids found in the marine mussel Mytilus edulis. ${ }^{29}$

It is often possible to employ catalytic amounts of chiral protonating agents, provided that an appropriate stoichiometric proton source can be identified. For this purpose, a number of chiral organic catalyts have been employed in various transformations. In order to achieve a heterocycle reduction/protonation strategy analogous to that used by Donohoe and coworkers (Figure 3d), Rueping and coworkers ${ }^{31}$ were able reduce substituted quinolines enantioselectively (Figure 4a). Initial hydride reduction of annulated quinoline 25 by a Hantzch dihydropyridine (30) to form enamine $\mathbf{2 6}$ was followed by enantioselective protonation with a catalytic chiral BINOL-phosphoric acid (29). Terminal hydride reduction of iminium ion $\mathbf{2 7}$ yielded optically active tetrahydroquinoline $\mathbf{2 8}$ as an 8:1 mixture of trans-and cisdiastereomers with the diastereomers formed in $94 \%$ and $99 \%$ enantiomeric excess, respectively. Up to $84 \%$ yield and $85 \%$ enantiomeric excess could be obtained for monosubstituted quinolines using the optimal reaction conditions.

In a mechanistically different approach, Cheon and Yamamoto ${ }^{32}$ used a related BINOL $N$ triflyl thiophosphoramide catalyst (33) to directly protonate cyclic silyl enol ethers (e.g., $\mathbf{3 1} \rightarrow$ 32, Figure 4b). Regeneration of the chiral proton source was made possible by using phenol as the achiral proton source. With this system, yields up to $99 \%$ and enantiomeric excesses up to $90 \%$ were achieved. The highest levels of enantioselectivity were obtained with substrates 
bearing an aryl substituent at the $\alpha$-position and 7-membered rings performed somewhat better than 6-membered rings. Comparable selectivity and yield could be obtained with catalyst loadings as low as $0.05 \mathrm{~mol} \%$. In control experiments, the protonation reaction did not proceed without an achiral proton source, even with stoichiometric chiral Brønsted acid. This observation led to a proposed mechanism that involves pre-association of the chiral and achiral proton sources to form an oxonium ion pair (34) that then protonates the silyl enol ether substrate.

A common approach to enantioselective protonation employs a lithium enolate, catalytic chiral proton source, and stoichiometric achiral proton source. These lithium enolates are often prepared from the corresponding enol silanes. Reports of amino acids as catalytic proton sources have appeared in recent literature. Yanagisawa and coworkers ${ }^{33}$ employed commercially available dipeptide 35 (Figure $4 \mathrm{c}$ ) for the protonation of a lithium enolates of tetralones and cyclohexanones. The catalytic proton source is regenerated through proton transfer from 2,6-di-t-butyl-4-methylphenol (BHT). The steric bulk of the phenol is important to suppressing background enolate protonation through a non-selective pathway. The structure of the chiral proton donor is very specific to the success of the reaction because isomeric dipeptide aspartame afforded racemic product. Enolates generated from deprotonation of racemic ketone 15 with lithium diisopropylamide could also be protonated with comparable yield and enantioselectivity using this system.

In separate work from Yanagisawa and coworkers, ${ }^{34,35}$ enol silanes were found to react to form ketones in the presence of the complex of AgF and BINAP (39, Figure 4d) with methanol as a proton donor. Excellent enantiomeric excesses were reported for a variety of cyclic ketones. Cyclohexanone-derived enol silanes yielded significantly higher e.e. products than tetralonederived enol ethers. The highest levels of e.e. (up to 99\%) were found for $\alpha$ -

arylcyclohexanones. Although the fine mechanistic details have not been elucidated, one possibility is that the chiral Lewis acidic Ag・BINAP complex binds methanol to generate a potent chiral Brønsted acid capable of protonating the latent enolate. Taking the fluoride activation component into account, aggregate complex $\mathbf{3 7}$ was proposed as a potential intermediate leading to the observed enantiomer of product ketone.

Levacher and coworkers ${ }^{36}$ have reported that catalytic amounts of Cinchona alkaloid derivatives can be coupled to latent hydrogen fluoride sources to achieve enantioselective protonation of silyl enol ethers of 1-indanones and 1-tetralones (e.g., $\mathbf{4 0} \rightarrow \mathbf{4 2}$, Figure 4e). The hydrogen fluoride, generated in situ from benzoyl fluoride and ethanol, is presumed to interact with the alkaloid-derived catalyst to form a cinchonium fluoride. The fluoride anion of this active catalyst then activates the silyl group to facilitate proton delivery from the ammonium cation (e.g., 41), through a proposed transition state reminiscent of the Ag・BINAP protonation protocol described above. The optimal alkaloid catalyt was (DHQ) $2(\mathrm{AQN})$, a common ligand for Sharpless' enantioselective dihydroxylation reactions.37 Yields up to $86 \%$ and enantiomeric excesses up to $92 \%$ were reported. Protonation of cyclohexanone-derived enol silanes gave moderate enantiomeric excess. Later work revealed that carboxylic acids (e.g., citric acid) could be used directly with the alkaloid-derived catalysts to carry out the enol silane protonation.38 Enantioselectivity for this variant of the reaction was moderately diminished, however (e.e. up to $75 \%$ ). The use of chiral carboxylic acids revealed a moderate, but measureable, influence of both the proton donor and the catalyst on enantioselection, with the catalyst influence apparently dominating.

In a series of reports by Deng and coworkers, ${ }^{39,40}$ bifunctional Cinchona alkaloid-derivatives (e.g., 21, 49-52, Figure 4f) were reported to catalyze tandem asymmetric conjugate addition/ protonation reactions of cyclic and acyclic $\alpha$-cyanoketone nucleophiles (e.g., 43 and $\mathbf{4 6}$ ) to 2chloroacrylonitrile (44). The Cinchona alkaloid derivative is proposed to serve two functions: 
activating the Michael acceptor for addition and serving as the chiral Brønsted acid for the protonation of the nitrile-stabilized carbanion intermediate. The postulated stereochemical model involves a network of hydrogen bonding interactions, and it was found that modification of this network by masking the phenolic hydroxyl group and introducing a thiourea moiety led to preferential formation of the diastereomeric products in excellent e.e. By virtue of this discovery, all four stereoisomeric products of $\mathbf{4 5}$ could be prepared with high degrees of diastereo- and enantioselectivity. This method was applied to an enantioselective formal synthesis of (-)-manzacidin A (48) via the intermediate thioester 47, prepared in $93 \%$ e.e. from $\alpha$-cyanothioester $\mathbf{4 6}$ and 2-chloroacrylonitrile (44). A very similar transformation was reported by $\mathrm{Wu}$ and coworkers ${ }^{41}$ for the conjugate addition of thiophenol to an $\alpha$-substituted acrylamide with subsequent enantioselective protonation in up to $60 \%$ e.e.

Organic catalysts other than Cinchona alkaloids have shown promise for catalytic asymmetric protonation as well. Tan and coworkers ${ }^{42}$ used thiols and phosphine oxides as nucleophiles for 1,4-addition into various phthalimidoacrylates and itaconimides (e.g., $\mathbf{5 3} \rightarrow \mathbf{5 5}$, Figure $4 \mathrm{~g}$ ). Subsequent protonation of the intermediate enolates by the conjugate acid of chiral bicyclic guanidine catalyst 54 gave up to $99 \%$ yield and $94 \%$ enantiomeric excess. Interestingly, the presence of water and relatively acidic thiophenols did not lead to appreciable amounts of nonselective enolate quenching. Kinetic isotope effects were explored with $\mathrm{D}_{2} \mathrm{O}$ and a primary kinetic isotope effect of 1.5 was found, consistent with cleavage or formation of a bond containing $\mathrm{H}$ or $\mathrm{D}$ in the rate-determining step.

An unusual technique to generate an enol in situ was reported by Hénin, Muzart and coworkers. ${ }^{43,44}$ In this work, a racemic $\alpha$-quaternary ketone (e.g., 56, Figure $4 \mathrm{~h}$ ) was exposed to laser photolysis at $366 \mathrm{~nm}$. This irradiation caused a Norrish type II fragmentation $(\mathbf{5 6} \rightarrow \mathbf{5 7})$ to occur with concomitant loss of isobutylene. The enol (57) then undergoes enantioselective transformation to the corresponding ketone (15) in the presence of amino alcohol 58. Although high e.e. was realized for this dealkylative protonation in some cases, yields were low largely due to undesired fragmentation reactions initiated by the photochemical irradiation and decomposition of the resulting radical intermediates. This method represents one of the few enantioselective stereoablative transformations ${ }^{45}$ involving destruction of a quaternary stereocenter.

A variety of transformations catalyzed by planar-chiral heterocycles involve protonation as the enantiodetermining step. In one recent example from Fu and coworkers, ${ }^{46}$ catalyst $\mathbf{6 0}$ (Figure 4i) was found to promote the addition of $\mathrm{HN}_{3}$ to ketenes (e.g., 59). The resulting acyl azides (e.g., 62) then underwent Curtius rearrangement at elevated temperatures. The carbamate products (e.g., 63) were isolated with up to $97 \%$ e.e., presumably from enantioselective protonation of the amide enolate intermediate (61). Sterically large ketenes performed best since less encumbered ketenes suffered from rapid uncatalyzed background reaction even at cryogenic temperatures. In control experiments, hydrazoic acid was found to be readily deprotonated by planar-chiral heterocycle catalyst $\mathbf{6 0}$. Since the reaction also proceeded with higher selectivity at low substrate concentration, a mechanism involving an ion pair $\mathbf{6 1}$ was proposed. This behavior of the catalyst as a chiral Brønsted acid rather than the more typical role of these catalysts as Lewis bases ${ }^{47}$ is somewhat unusual, but also serves as a testament to the privileged nature of these catalysts. ${ }^{48}$

Although the particular example in Figure 4i is thought to occur through a protonated catalyst molecule as the proton donor, other similar transformations may proceed through a different mechanism (see the following section), however in both examples the lack of a nonlinear relationship ${ }^{49}$ between catalyst e.e. and product e.e. suggests that only one catalyst molecule is involved in the enantioselective step and therefore a hybrid mechanism is unlikely.

Regardless of which of these two mechanistic hypotheses is operative, the transformation 
remains an enantioselective protonation process. An understanding of the mechanistic details, however, is helpful in order to understand the stereochemical control elements important to the success of the transformation.

\section{Enantioselective Protonation by Means of Chiral Brønsted Base}

The most common form of chiral Brønsted base employed in enantioselective protonations has been chiral metal enolates. To circumvent the challenge of stereoselective generation of acyclic enolates, the majority of methods have focused on cyclic enolate precursors. A variety of techniques for enolate generation have been employed including simple deprotonations, pericyclic reactions, decarboxylations, and dehalogenations. The synthesis of the specific enolate precursor required for a protonation protocol (e.g., enol silane, acrylate, or $\beta$-ketoester) may determine how useful the method is for the preparation of specific target molecules. Although several successful chiral Brønsted base protonation protocols have been developed, substrate scope remains a significant problem. Especially lacking are general methods capable of protonation of acyclic enolates with high selectivity.

Planar-chiral heterocycle catalysts have also been employed in the generation of chiral Brønsted base intermediates for enantioselective protonation. Fu and coworkers ${ }^{50}$ employed azaferrocene catalyst 65 (Figure 5a). In the proposed reaction mechanism the heterocycle catalyst reacts with a ketene (e.g., 64) to form a zwitterionic chiral enolate intermediate (66). Subsequent protonation of the enolate by methanol or an achiral pyridinium cocatalyst then forms an acylferrocenium ion (e.g., 67) that goes on to form an enantioenriched ester (e.g., 68). Evidence for this distinct reaction mechanism included the fact that catalyst 65 did not appreciably deprotonate methanol in solution. ${ }^{51}$ However, in the case above (Figure 4i) the catalyst $\mathbf{6 0}$ does readily deprotonate hydrazoic acid. The remarkable ability of these Lewis base catalysts to achieve enantioselective protonation through two different mechanisms highlights the importance of understanding the details of the reaction pathways. The preliminary mechanistic evidence does not always provide sufficient information to determine the operative mechanism, however. ${ }^{52}$

Other organic molecules have been employed for the generation of chiral enolates as well. For example, Rovis and coworkers ${ }^{53}$ found that treatment of $\alpha, \alpha$-dichloroaldehydes such as 69 (Figure 5b) with enantiopure triazolium salt catalyst $\mathbf{7 0}$ in the presence of a stoichiometric phenoxide base and phenol led to $\alpha$-chloroaldehydes with high enantiomeric excess. The proposed path of the reaction involves addition of the carbene conjugate base of catalyst $\mathbf{7 0}$ to the aldehyde substrate. Subsequent elimination of $\mathrm{HCl}$ then generates a zwitterionic enolate intermediate (71) that is subject to protonation to form enantioenriched aldehyde $\mathbf{7 2}$. The precise structure of the putative enolate is a matter of conjecture since a number of different strain elements are likely in competition in this intermediate. Nonetheless, this method represents a very valuable technique for the preparation of useful, but synthetically challenging, chiral synthons.

In a separate application of carbene catalysis, Scheidt and coworkers ${ }^{54}$ found that homoenolate equivalents (e.g., 75, Figure 5c), generated in situ from enals such as $\mathbf{7 3}$, could undergo enantioselective protonation when chiral triazolium catalyst $\mathbf{7 4}$ was employed. Ester products (e.g., 76) were obtained in up to 58\% yield and up to 59\% e.e., although competitive formation of overoxidized enoate byproduct 77 complicated the reaction. Although the degree of enantioselectivity observed for this transformation was modest, this represents a significant advance in the type of proton acceptor that can be used for enantioselective protonation reactions since the site of protonation is relatively distant from the chiral control element.

Metal-catalyzed 1,4-additions of organoboranes or organosilanes to enone systems have proven to be a popular strategy for generating chiral enolates for asymmetric protonation. These 
reactions can employ neutral or cationic rhodium complexes. Arylboron, arylstannane, arylsilicon, or arylzinc (and in some cases, vinylboron) reagents can be used for coupling, but yields and enantioselectivities can depend on the type of organometallic reagent chosen. Atropisomeric bis(phosphine) ligands such as BINAP (39) and its analogues give excellent enantioselectivities for simple $\beta$-unsubstituted enone substrates. Diverse proton sources have been used to enable the formation of $\alpha$-stereocenters. The optimal proton source varies from system to system, but phenols and other low- $\mathrm{p} K_{\mathrm{a}}$ proton sources are common. Recent examples have mainly explored simple $\beta$-unsubstituted systems, so issues of diastereoselectivity in the generation of multiple stereocenters have not been fully addressed.

Frost and coworkers ${ }^{55}$ explored Rh-catalyzed additions of organotrifluoroborate salts to itaconate substrates with water as the stoichiometric proton donor (e.g., $78 \rightarrow \mathbf{7 9} \rightarrow \mathbf{8 0}$, Figure 6a). The metal-to-ligand ratio was found to be important in the selectivity of the reaction. Equimolar amounts of metal and ligand gave racemic product in high yield, but 2.2 equivalents of ligand relative to metal gave product in $82 \%$ ee and 56\% yield. Interestingly, high reaction temperature was found to be an important factor in this reaction; racemic product was obtained when the reaction was carried out at 60 or $80^{\circ} \mathrm{C}$ instead of $110^{\circ} \mathrm{C}$. In related work, Frost showed that organosiloxanes are also viable nucleophilic components for 1,4-addition, but such systems give poor yields and low enantioselectivity. ${ }^{56}$ In a separate system involving aryl boronic acid organometallic components in microwave reactors, Frost and coworkers ${ }^{57}$ examined whether a chiral proton source affected the stereochemical outcome of the reaction. When $(R)_{-},(S)$-, or racemic-BINOL was used with a Rh•BINAP catalyst, the yields and enantioselectivities were comparable in each of the three scenarios. This suggests that the chirality of the bis(phosphine) ligand is the dominant stereodetermining factor.

Sibi and coworkers ${ }^{58}$ also investigated rhodium-catalyzed conjugate addition-protonation as a method for accessing $\beta^{2}$-amino esters from enoates (e.g., $\mathbf{8 1} \rightarrow \mathbf{8 3}$, Figure 6a). Reaction screening revealed that $(S)$-DifluorPhos ligand $(\mathbf{8 2})$ and phthalimide proton donor provided the best enantioselectivity. The best substrate provided $95 \%$ yield and $91 \%$ ee. Notably, lower temperatures could be used to increase product e.e.

Hayashi and coworkers ${ }^{59}$ reported a related conjugate addition/protonation sequence for an analogous diphenylphosphinylallene (e.g., 84) system with a cationic dimeric rhodium $\mu_{2}$ hydroxide catalyst (Figure 6a). Temperature did not appear to have an effect on enantioselectivities, but the choice of THF as solvent instead of dioxane improved enantioselectivities by more than $20 \%$. Minimal isomerization of the $\beta, \gamma$-unsaturated products (e.g., 85) was observed under the reaction conditions, and excellent yields (up to 97\%) and enantioselectivities (up to 98\%) were obtained. In this case, the arylboronic acid appears to be acting as the proton source based on an NMR experiment.

Sodeoka and coworkers ${ }^{60}$ found that this conjugate addition/protonation sequence is viable with palladium complexes and heteroatom nucleophiles as well $(\mathbf{8 6} \rightarrow \mathbf{8 7}$, Figure $6 \mathrm{~b})$. A bimetallic palladium $\mu_{2}$-hydroxide complex effected the conjugate addition of aniline derivatives to acrylamides. With BINAP ligand, the enolate intermediate was protonated enantioselectively to give product 87 in $80 \%$ yield and $94 \%$ ee. Although this is a promising result, the substrate scope has not been fully investigated.

Genet, Darses, and coworkers ${ }^{61,62}$ sought to make $\alpha$-amino esters using a similar conjugate addition/protonation strategy (e.g., $\mathbf{8 8} \rightarrow \mathbf{9 3}$, Figure 6 c). In this system, boronic esters, boronic acids, and organosilanes did not provide adequate conversion or enantioselectivity.

Organostannanes, however, were reactive under these conditions. In the screening of various proton sources, phenol derivatives appeared to provide the best enantioselectivity. Guaiacol (2-methoxyphenol) was determined to be the best proton donor, giving products with up to 
91\% yield and $88 \%$ ee. Higher temperatures and optimized solvents (such as toluene or dioxane) increased conversion and enantioselectivity. As in Frost's work, the ratio of metal to ligand did have a notable effect on the enantioselectivity of the reaction, with 2.2 equivalents of chiral ligand relative to metal being optimal. Chiral proton sources were also added to see if the chiral ligand or chiral proton source was the dominant selectivity factor. Studies with $(R)$-BINAP and both enantiomers of BINOL provided identical enatiomeric excesses, implying that the chirality of the enolate is dominant, as in the reports by Frost.

Mechanistic studies showed that the hydrogen on the enamide was essential for reactivity. Deuterium labeling studies showed that the reaction did not proceed via a direct protonation of the rhodium enolate (89); in a substrate with a labeled carbamate proton, deuterium incorporation at the $\alpha$-carbon was high (41\%). Based on this observation, a mechanism involving sequential conjugate addition, $\beta$-hydride elimination from the carbamate $(\mathbf{9 0} \rightarrow$ 91), and intramolecular hydride transfer to the $\alpha$-carbon $(\mathbf{9 1} \rightarrow \mathbf{9 2})$ was proposed. This proposed reaction pathway was supported by DFT calculations with the B3LYP/BII level of theory. ${ }^{62}$ In light of these mechanistic insights, a more electron-poor bis(phophine) ligand was chosen to facilitate the postulated $\beta$-hydride elimination step, and DifluorPhos (82) was experimentally shown to give higher enantiomeric excesses.

A related conjugate addition/enantioselective protonation strategy consisted of a FriedelCrafts-type addition of pyrroles to $\alpha$-substituted acrylates (e.g., $94 \rightarrow \mathbf{9 7}$, Figure $6 \mathrm{~d}$ ). ${ }^{63}$ Sibi and coworkers employed $\mathrm{Zn}\left(\mathrm{NTf}_{2}\right)$ complexed with chiral dbfox ligand $\mathbf{9 5}$ as a Lewis acid catalyst to achieve up to $98 \%$ yield and up to $98 \%$ e.e. The enolate intermediate (96) generated from the Friedel-Crafts reaction can be quenched by a proton from the pyrrole fragment to give a chiral product. The isoxazolidinone auxiliary is believed to be critical for improving reactivity and providing greater enolate control during the course of the reaction.

A distinct method of accessing a chiral metal enolate is the use of a pericyclic reaction. In the case of the Nazarov cyclization, the enantioselective reaction would not only generate a new stereocenter, but also a new five-membered ring. However, until recently enantioselective variants of this classical reaction were unknown. In studies directed toward the development of a general enantioselective Nazarov cyclization, Trauner and coworkers ${ }^{64}$ discovered that high e.e. cyclopentenone products could be synthesized from electronically activated substrates such as dienone 98 in the presence of Sc•PYBOX complex 99 (Figure 7a). Interestingly, this enantioselective protonation protocol requires no external proton source. Unfortunately, in cases where a second stereocenter would be generated at the $\beta$-carbon (i.e., 103 where $R \neq H$ ), low diastereoselectivity was observed. This was interpreted as a result of low stereoselectivity in the electrocyclization step $(\mathbf{1 0 0} \rightarrow \mathbf{1 0 1})$ followed by high selectivity in the protonation step $(101 \rightarrow 102)$.

A different strategy for accessing chiral metal enolates is decarboxylation of $\beta$-ketoesters. The nature of the ester is typically important to enable enolate formation. In the case of palladiumcatalyzed protonation reactions, benzyl and allyl esters have been the most common enolate precursors. The first examples of these reactions, developed extensively by Hénin, Muzart, and coworkers, 65 employed an initiation step such as Pd-mediated hydrogenolysis to generate an achiral enol (or enolate)66,6768 intermediate that was subsequently protonated with a chiral Brønsted acid (typically an amino alcohol). The results of these studies showed a strong dependence on the specific reaction conditions (e.g., temperature and source of palladium on carbon). More recently, this strategy has been refined to instead generate a chiral Pd-enolate intermediate from allyl $\beta$-ketoester substrates (e.g., 104 and 107, Figure 7c) in the hopes that chirality at the metal might impart improved stereocontrol in the protonation step and obviate the need for heterogeneous Pd catalysts. Stoltz and coworkers ${ }^{69}$ found that the catalyst derived from $\mathrm{Pd}(\mathrm{OAc})_{2}$ and phosphinooxazoline (PHOX) ligand 105 was capable of achieving 
enantioselective protonation in the presence of formic acid and molecular sieves (MS). High enantiomeric excesses were obtained for a range of cyclic ketone products including tetraloneand cyclohexanone-derived ketones (e.g., 106 and 109). Attempts to track the source of the proton incorporated in the product with isomeric mono-deuterated formic acids $\left(\mathrm{DCO}_{2} \mathrm{H}\right.$ and $\mathrm{HCO}_{2} \mathrm{D}$ ) led to inconclusive results: with $\mathrm{DCO}_{2} \mathrm{H}$ the formyl deuteron was not incorporated into the product, but with $\mathrm{HCO}_{2} \mathrm{D}$ only $35 \% \mathrm{D}$ incorporation was observed in the product, suggesting that another unidentified proton donor is also participating in the reaction. The heterogeneous additive (MS) in the reaction is important to the selectivity for protonation in preference to the competitive allylation reaction also catalyzed by the Pd•PHOX complex, ${ }^{70}$, 71,72 and the specific amount required varied somewhat depending on the substrate. To address this issue, a related homogeneous protonation system was developed by Stoltz and coworkers ${ }^{73}$ employing Meldrum's acid (108) as the achiral proton donor and a Pd•PHOX catalyst. Similar substrate scope and enantioselectivity was observed with this system, but without the need to optimize the amounts of additives to achieve optimal results. Initial mechanistic studies found zero-order kinetic dependence in substrate, which suggests a fast initial reaction between catalyst and substrate and a slow subsequent step, presumably either decarboxylation or protonation of the putative enolate intermediate common to both the protonation and allylic alkylation reactions with this catalyst system. ${ }^{74}$ However, in the protonation reaction opposite enolate facial preference was observed for tetralone- and cyclohexanone-derived enolates, whereas in the alkylation reaction consistent enantiofacial selectivity was observed. ${ }^{70,71,72}$ This unexpected result suggests a substantial difference in the bond-forming portion of the mechanism.

Kanai, Shibasaki, and coworkers ${ }^{75}$ recently reported the use of a chiral gadolinium complex for the enantioselective protonation of Gd-enolates generated in situ (Figure 7d). The Gdenolates were accessed by two means: transmetallation from enol silane (e.g., 14) or conjugate addition of cyanide to $N$-acryloyl pyrroles (e.g., 111). Based on optimization studies, a polymetallic enolate intermediate was proposed. The optimal ligand-to-metal ratio is consistent with a 5:6 complex. The lack of reactivity in the absence of ligand $\mathbf{1 1 0}$ was also interpreted as the necessity of polynuclear Gd complexes for the success of the reaction. Kinetic studies also suggested that the reaction proceeds via transmetallation from $\mathrm{Si}$ to $\mathrm{Gd}$, and that this step is rate-limiting. 2,6-Dimethylphenol was the preferred proton source, providing indanone and tetralone products in up to $99 \%$ yield and up to $88 \%$ e.e. The same $\mathrm{Gd} \cdot \mathbf{1 1 0}$ complex was also effective for the conjugate addition/protonation of cyanide to $N$-acryloyl pyrroles (e.g., $111 \rightarrow$ 112). In this case, hydrogen cyanide was the preferred proton source. This transformation was capable of producing $N$-acyl pyrroles in up to $99 \%$ yield and up to $91 \%$ ee. In the case of product 112, recrystallization could be carried out to provide a $74 \%$ yield of material with $>99 \%$ ee. The versatility of this polynuclear Gd catalyst to effect protonation of considerably different enolate intermediates is important to the prospect of a general system for enolate protonation.

\section{Enantioselective Hydrogen Atom Transfer}

An analogous strategy for arriving at protonation products is to exploit radical chemistry with a terminal H-atom abstraction step. Although not explicitly an enantioselective protonation reaction, many of the same challenges apply when manipulating a hydrogen atom. A recent example of this tactic by Sibi and coworkers ${ }^{76,77}$ has led to new methods for synthesizing $\beta$ amino acid derivatives (Figure 8). By employing a Lewis acid complex formed from $\mathrm{MgI}_{2}$ and bis(oxazoline) ligand 113, tributyltin hydride, and triethylborane $/ \mathrm{O}_{2}$, alkyl radicals (from alkyl halides) can undergo radical conjugate addition followed by enantioselective hydrogen atom transfer to afford optically active products (e.g., 114) in up to 95\% yield and up to $98 \%$ e.e. The reaction is believed to proceed through a bidentate chelate of the substrate to the chiral magnesium complex. The process can be made catalytic in Lewis acid without a significant 
effect on yield, but product e.e. suffered greatly in some cases due to a rapid uncatalyzed background reaction. These reactions have the benefit of being essentially neutral as opposed to the acidic or basic conditions required for the transformations described above.

\section{Conclusion}

From the enantioselective enzymatic protonation reactions of nature to the variety of techniques for achieving enantioselective protonation in laboratories, a great deal of energy has been dedicated to understanding this deceptively simple transformation. Despite the many systems reported to date, the search for a highly efficient system with a broad scope continues. Moreover, although many of the key parameters needed to create a successful system have been enumerated here and elsewhere, the current level of mechanistic understanding in nearly all of the enantioselective protonation reactions reported to date remains relatively immature. Nonetheless, these useful transformations allow the synthesis of valuable chiral materials including natural products like $\alpha$ - and $\beta$-amino acids. Given these preliminary successes and improved understanding of the underlying mechanisms, enantioselective protonation reactions should continue to rise to prominence as an important tool for synthetic organic chemistry.

\section{Acknowledgments}

We thank the National Institutes of Health, National Institute of General Medical Sciences (grant number R01GM080269-01), Eli Lilly (predoctoral fellowship to J.T.M.), Amgen, Abbott Laboratories, Boehringer Ingelheim, Merck, Bristol-Myers Squibb, and the California Institute of Technology for financial support.

\section{References}

1. Mohr JT, Krout MR, Stoltz BM. Natural products as inspiration for the development of asymmetric catalysis. Nature 2008;455:323-332. [PubMed: 18800131]

2. Fehr C. Enantioselective protonation of enolates and enols. Angew Chem, Int Ed Engl 1996;35:25662587.

3. Yanagisawa A, Ishihara K, Yamamoto H. Asymmetric protonations of enol derivatives. Synlett 1997:411-420.

4. Eames J, Weerasooriya N. Recent advances into the enantioselective protonation of protostereogenic enol derivatives. Tetrahedron: Asymmetry 2001;12:1-24.

5. Duhamel L, Duhamel P, Plaquevent JC. Enantioselective protonations: Fundamental insights and new concepts. Tetrahedron: Asymmetry 2004;15:3653-3691.

6. Yanagisawa, A.; Yamamoto, H. Comprehensive Asymmetric Catalysis. Jacobsen, EN.; Pfaltz, A.; Yamamoto, H., editors. Vol. III. Springer; 1999. p. 1295-1306.

7. Yanagisawa, A.; Yamamoto, H. Comprehensive Asymmetric Catalysis. Jacobsen, EN.; Pfaltz, A.; Yamamoto, H., editors. Springer; 2004. p. 125-132.

8. Blanchet J, Baudoux J, Amere M, Lasne M-C, Rouden J. Asymmetric malonic and acetoacetic acid syntheses - a century of enantioselective decarboxylative protonations. Eur J Org Chem 2008:54935506.

9. Fehr C. Catalytic enantioselective tauromerization of isolated enols. Angew Chem, Int Ed 2007;46:7119-7121.

10. Miyamoto K, Ohta H. Purification and properties of a novel arylmalonate decarboxylase from Alcaligenes bronchisepticus KU 1201. Eur J Biochem 1992;210:475-481. [PubMed: 1459132]

11. Matoishi K, Ueda M, Miyamoto K, Ohta H. Mechanism of asymmetric decarboxylation of $\alpha$-aryl- $\alpha$ methylmalonate catalyzed by arylmalonate decarboxylase originated from Alcaligenes bronchisepticus. J Mol Catal B: Enzym 2004;27:161-168.

12. Hansch C, Leo A, Taft RW. A survey of Hammett substituent constants and resonance and field parameters. Chem Rev 1991;91:165-195. and references therein. 
13. Nakasako M, Obata R, Okubo R, Nakayama S, Miyamoto K, Ohta H. Crystallization and preliminary $\mathrm{X}$-ray diffraction experiments of arylmalonate decarboxylase from Alcaligenes bronchisepticus. Acta Crystallogr Sect F 2008;64:610-613.

14. Kuettner EB, Keim A, Kircher M, Rosmus S, Strater N. Active site mobility revealed by the crystal structure of arylmalonate decarboxylase from Bortadella bronchiseptica. J Mol Biol 2008;377:386394. [PubMed: 18258259]

15. Terao Y, Ijima Y, Miyamoto K, Ohta H. Inversion of the enantioselectivity of arylmalonate decarboxylase via site-directed mutation based on the proposed reaction mechanism. J Mol Catal B: Enzym 2007;45:15-20.

16. Ijima Y, Matoishi K, Terao Y, Doi N, Yanagawa H, Ohta H. Inversion of the enantioselectivity of asymmetric biocatalytic decarboxylation by site-directed mutagenesis based on the reaction mechanism. Chem Commun 2005:877-879.

17. Terao Y, Miyamomoto K, Ohta H. Improvement of the activity of arylmalonate decarboxylase by random mutagenesis. Appl Microbiol Biotechnol 2006;73:647-653. [PubMed: 16865343]

18. Miyamoto K, Hirokawa S, Ohta H. Conversion of $\alpha$-methyltropate to optically active $\alpha$ phenylpropionate by tropate-degrading Rhodococcus sp. KU1314. J Mol Catal B: Enzym 2007;46:14-19.

19. Matsumoto K, Tsutsumi S, Ihori T, Ohta H. Enzyme-mediated enantioface-differentiating hydrolysis of $\alpha$-substituted cycloalkanone enol esters. J Am Chem Soc 1990;112:9614-9619.

20. Hirata T, Shimoda K, Kawano T. Asymmetric hydrolysis of enol esters with two esterases from Marchantia polymorpha. Tetrahedron: Asymmetry 2000;11:1063-1066.

21. Sakai T, Matsuda A, Tanaka Y, Korenaga T, Ema T. The effect of temperature on the lipase-catalyzed asymmetric protonation on 1-acetoxy-2-methylcyclohexene giving $(R)$-2-methylcyclohexanone. Tetrahedron: Asymmetry 2004;15:1929-1932.

22. Vedejs E, Kruger AW, Suna E. Enantioselective enolate protonation: Matching chiral aniline and substrate acidity. J Org Chem 1999;64:7863-7870.

23. Kim BM, Kim H, Kim W, Im KY, Park JK. Asymmetric protonation of ketone enolates using chiral $\beta$-hydroxyethers: Acidity-tuned enantioselectivity. J Org Chem 2004;69:5104-5107. [PubMed: 15255744]

24. Boyd E, Coumbarides GS, Eames J, Hay A, Jones RVH, Stenson RA, Suggate MJ. Reversal of enantioselectivity on protonation of enol(ate)s derived from 2-methyl-1-tetralone using $C_{2}$ symmetric sulfonamides. Tetrahedron Lett 2004;45:9465-9468.

25. Coumbarides GS, Eames J, Ghilagaber S, Suggate MJ. Investigations into the enantioselective Cprotonation of protostereogenic enolate(s) derived from $N, N^{\prime}$-diisopropyl-2-phenylpropanamide using suicide $C$-based proton sources. Tetrahedron Lett 2004;45:9469-9474.

26. Coumbarides GS, Eames J, Scheuermann JEW, Sibbons KF, Suggate MJ, Watkinson M. Enantioselective protonation of a lithium enolate derived from 2-methyl-1-tetralone using chiral sulfonamides. Bull Chem Soc Jpn 2005;78:906-909.

27. Amere M, Lasne MC, Rouden J. Highly enantioselective decarboxylative protonation of $\alpha$ aminomalonates mediated by thiourea Cinchona alkaloid derivatives: Access to both enantiomers of cyclic and acyclic $\alpha$-aminoacids. Org Lett 2007;9:2621-2624. [PubMed: 17579443]

28. Seitz T, Baudoux J, Bekolo H, Cahard D, Plaquevent JC, Lasne MC, Rouden J. Organocatalyzed route to enantioenriched pipecolic esters: Decarboxylation of an aminomalonate hemiester. Tetrahedron 2006;62:6155-6165.

29. Carbery DR, Donohoe TJ. Enantiopure oxazolidinones as chiral acids in the asymmetric protonation of N-Boc pyrrole derived enolates. Chem Commun 2004:722-723.

30. Donohoe TJ, Freestone GC, Headley CE, Rigby CL, Cousins RPC, Bhalay G. Enantioselective partial reduction of 2,5-disubstituted pyrroles via a chiral protonation approach. Org Lett 2004;6:30553058. [PubMed: 15330586]

31. Rueping M, Theissmann T, Raja S, Bats JW. Asymmetric counterion pair catalysis: An enantioselective Brønsted acid-catalyzed protonation. Adv Synth Catal 2008;350:1001-1006.

32. Cheon $\mathrm{CH}$, Yamamoto $\mathrm{H}$. A Brønsted acid catalyst for the enantioselective protonation reaction. J Am Chem Soc 2008;130:9246-9247. [PubMed: 18582053] 
33. Mitsuhashi K, Ito R, Arai T, Yanagisawa A. Catalytic asymmetric protonation of lithium enolates using amino acid derivatives as chiral proton sources. Org Lett 2006;8:1721-1724. [PubMed: 16597150]

34. Yanagisawa A, Touge T, Arai T. Enantioselective protonation of silyl enolates catalyzed by a Binap•AgF complex. Angew Chem, Int Ed 2005;44:1546-1548.

35. Yanagisawa A, Touge T, Arai T. Asymetric protonation of silyl enolates catalyzed by chiral phosphine-silver(I) complexes. Pure Appl Chem 2006;78:519-523.

36. Poisson T, Dalla V, Marsais F, Dupas G, Oudeyer S, Levacher V. Organocatalytic enantioselective protonation of silyl enolates mediated by Cinchona alkaloids and a latent source of HF. Angew Chem, Int Ed 2007;46:7090-7093.

37. Becker H, Sharpless KB. A new ligand class for the asymmetric dihydroxylation of olefins. Angew Chem, Int Ed Engl 1996;35:448-451.

38. Poisson T, Oudeyer S, Dalla V, Marsais F, Levacher V. Straightforward organocatalytic enantioselective protonation of silyl enolates by means of Cinchona alkaloids and carbxylic acids. Synlett 2008;16:2447-2450.

39. Wang Y, Liu X, Deng L. Dual-function Cinchona alkaloid catalysis: Catalytic asymmetric tandem conjugate addition-protonation for the direct creation of nonadjacent stereocenters. J Am Chem Soc 2006;128:3928-3930. [PubMed: 16551098]

40. Wang B, Wu F, Wang Y, Liu X, Deng L. Control of diastereoselectivity in tandem asymmetric reactions generating nonadjacent stereocenters with bifunctional catalysis by Cinchona alkaloids. J Am Chem Soc 2007;129:768-769. [PubMed: 17243806]

41. Li B-J, Jiang L, Liu M, Chen Y-C, Ding L-S, Wu Y. Asymmetric Michael addition of arylthiols to $\alpha, \beta$-unsaturated carbonyl compounds catalyzed by bifunctional organocatalysts. Synlett 2005:603606.

42. Leow D, Lin S, Chittimalla SK, Fu X, Tan CH. Enantioselective protonation catalyzed by a chiral bicyclic guanidine derivative. Angew Chem Int Ed 2008;47:5641-5645.

43. Hénin F, Muzart J, Pete JP, M'boungou-M'passi A, Rau H. Enantioselective protonation of a simple enol: Aminoalcohol-catalyzed ketonization of a photochemically produced 2-methylinden-3-ol. Angew Chem, Int Ed Engl 1991;30:416-418.

44. Hénin F, M'boungou-M'passi A, Muzart J, Pete JP. Photoreactivity of $\alpha$-tetrasubstituted arylketones: Production and asymmetric tautomerization of arylenols. Tetrahedron 1994;50:2849-2864.

45. Mohr JT, Ebner DC, Stoltz BM. Catalytic enantioselective stereoablative reactions: an unexploited approach to enantioselective catalysis. Org Biomol Chem 2007;5:3571-3576. [PubMed: 17971984]

46. Dai X, Nakai T, Romero JAC, Fu GC. Enantioselective synthesis of protected amines by the catalytic asymmetric addition of hydrazoic acid to ketenes. Angew Chem, Int Ed 2007;46:4367-4369.

47. Denmark SE, Beutner GL. Lewis base catalysis in organic synthesis. Angew Chem, Int Ed 2008;47:1560-1638.

48. Hodous BL, Fu GC. Enantioselective addition of amines to ketenes catalyzed by a planar-chiral derivative of PPY: Possible intervention of chiral Brønsted-acid catalysis. J Am Chem Soc 2002;124:10006-10007. [PubMed: 12188662]

49. Girard C, Kagan HB. Nonlinear effects in asymmetric synthesis and stereoselective reactions: Ten years of investigation. Angew Chem, Int Ed 1998;37:2922-2959.

50. Hodous BL, Ruble JC, Fu GC. Enantioselective addition of alcohols to ketenes catalyzed by a planarchiral azaferrocene: Catalytic asymmetric synthesis of arylpropionic acids. J Am Chem Soc 1999;121:2637-2638.

51. Wiskur SL, Fu GC. Catalytic asymmetric synthesis of esters from ketenes. J Am Chem Soc 2005;127:6176-6177. [PubMed: 15853315]

52. Schaefer C, Fu GC. Catalytic asymmetric couplings of ketones with aldehydes to generate enol esters. Angew Chem, Int Ed 2005;44:4606-4608.

53. Reynolds NT, Rovis T. Enantioselective protonation of catalytically generated chiral enolates as an approach to the synthesis of $\alpha$-chloroesters. J Am Chem Soc 2005;127:16406-16407. [PubMed: 16305222]

54. Maki BE, Chan A, Scheidt KA. Protonation of homoenolate equivalents generated by $N$-heterocyclic carbenes. Synthesis 2008:1306-1315. 
55. Moss RJ, Wadsworth KJ, Chapman CJ, Frost CG. Rhodium catalysed tandem conjugate additionprotonation: An enantioselective synthesis of 2-substituted succinic esters. Chem Commun 2004:1984-1985.

56. Hargrave JD, Herbert J, Bish G, Frost CG. Rhodium-catalysed addition of organotrialkoxysilanes to $\alpha$-substituted acrylic esters. Org Biomol Chem 2006;4:3235-3241. [PubMed: 17036111]

57. Frost CG, Penrose SD, Lambshead K, Raithby PR, Warren JE, Gleave R. Rhodium-catalyzed conjugate addition-enantioselective protonation: The synthesis of $\alpha, \alpha^{\prime}$-dibenzyl ethers. Org Lett 2007;9:2119-2122. [PubMed: 17465559]

58. Sibi MP, Tatamidani H, Patil K. Enantioselective rhodium enolate protonations. A new methodology for the synthesis of $\beta^{2}$-amino acids. Org Lett 2005;7:2571-2573. [PubMed: 15957893]

59. Nishimura T, Hirabayashi S, Yasuhara Y, Hayashi T. Rhodium-catalyzed asymmetric hydroarylation of diphenylphosphinylallenes with arylboronic acids. J Am Chem Soc 2006;128:2556-2557. [PubMed: 16492038]

60. Hamashima Y, Somei H, Shimura Y, Tamura T, Sodeoka M. Amine-salt-controlled, catalytic asymmetric conjugate addition of various amines and asymmetric protonation. Org Lett 2004;6:1861-1864. [PubMed: 15151433]

61. Navarre L, Darses S, Genet JP. Tandem 1,4-addition/enantioselective protonation catalyzed by rhodium complexes: Efficient access to $\alpha$-amino acids. Angew Chem, Int Ed 2004;43:719-723.

62. Navarre L, Martinez R, Genet JP, Darses S. Access to enantioenriched $\alpha$-amino esters via rhodiumcatalyzed 1,4-addition/enantioselective protonation. J Am Chem Soc 2008;130:6159-6169. [PubMed: 18396874]

63. Sibi MP, Coulomb J, Stanley LM. Enantioselective enolate protonations: Friedel-Crafts reactions with $\alpha$-substituted acrylates. Angew Chem Int Ed 2008;47:9913-9915.

64. Liang G, Trauner D. Enantioselective Nazarov reactions through catalytic asymmetric proton transfer. J Am Chem Soc 2004;126:9544-9545. [PubMed: 15291550]

65. Jamal Aboulhoda S, Hénin F, Muzart J, Thorey C, Behnen W, Martens J, Mehler T. Production of optically active ketones by a palladium-induced cascade reaction from racemic $\beta$-ketoesters. Tetrahedron: Asymmetry 1994;5:1321-1326.

66. Detalle JF, Riahi A, Steinmetz V, Hénin F, Muzart J. Mechanistic insights into the palladium-induced domino reaction leading to ketones from benzyl $\beta$-ketoesters: First characterization of the enol as an intermediate. J Org Chem 2004;69:6528-6532. [PubMed: 15387574]

67. Kukula P, Matousek V, Mallat T, Baiker A. Structural effects in the Pd-induced enantioselective deprotection-decarboxylation of $\beta$-ketoesters. Tetrahedron: Asymmetry 2007;18:2859-2868.

68. Kukula P, Matousek V, Mallat T, Baiker A. Enantioselective decarboxylation of $\beta$-keto esters with Pd/amino alcohol systems: Successive metal catalysis and organocatalysis. Chem-Eur J 2008;14:2699-2708.

69. Mohr JT, Nishimata T, Behenna DC, Stoltz BM. Catalytic enantioselective decarboxylative protonation. J Am Chem Soc 2006;128:11348-11349. [PubMed: 16939246]

70. Behenna DC, Stoltz BM. The enantioselective Tsuji allylation. J Am Chem Soc 2004;126:1504415045. [PubMed: 15547998]

71. Mohr JT, Behenna DC, Harned AM, Stoltz BM. Deracemization of quaternary stereocenters by Pdcatalyzed enantioconvergent decarboxylative allylation of racemic $\beta$-ketoesters. Angew Chem, Int Ed 2005;44:6924-6927.

72. Mohr JT, Stoltz BM. Enantioselective Tsuji allylations. Chem-Asian J 2007;2:1476-1491. [PubMed: 17935094]

73. Marinescu SC, Nishimata T, Mohr JT, Stoltz BM. Homogeneous Pd-catalyzed enantioselective decarboxylative protonation. Org Lett 2008;10:1039-1042. [PubMed: 18303896]

74. Keith JA, Behenna DC, Mohr JT, Ma S, Marinescu SC, Oxgaard J, Stoltz BM, Goddard WA III. The inner-sphere process in the enantioselective Tsuji allylation reaction with $(S)-t$-Buphosphinooxazoline (PHOX) ligands. J Am Chem Soc 2007;129:11876-11877. [PubMed: 17824701]

75. Morita M, Drouin L, Motoki R, Kimura Y, Fujimori I, Kanai M, Shibasaki M. Two methods for catalytic generation of reactive enolates promoted by a chiral poly Gd complex: Application to catalytic enantioselective protonation reactions. J Am Chem Soc. in press. 10.1021/ja9005018 
76. Sibi MP, Asano Y, Sausker JB. Enantioselective hydrogen atom transfer reactions: Synthesis of $N$ acyl- $\alpha$-amino acid esters. Angew Chem Int Ed 2001;40:1293-1296.

77. Sibi MP, Patil K. Enantioselective hydrogen atom transfer reactions: A new methodology for the synthesis of $\beta^{2}$-amino acids. Angew Chem Int Ed 2004;43:1235-1238. 

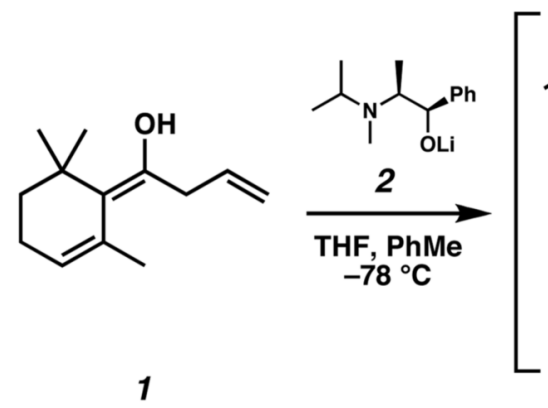<smiles></smiles>

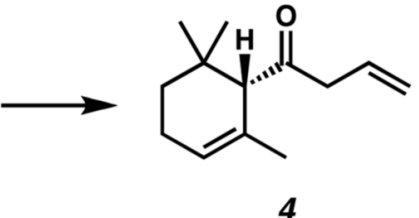

2 equiv $2,97 \%$ ee 0.5 equiv $2,83 \%$ ee

Figure 1. Enantioselective tautomerization of an isolated enol

Fehr ${ }^{9}$ demonstrated that enols in the presence of a chiral Lewis base may be transformed into enantioenriched ketones. This indicates a possible alternative mechanism for enantioselective protonation and suggests that sometimes these transformations may be better described as enantioselective tautomerization rather than protonation. (Reduced to $45 \%$ ) 
a) Enzymatic decarboxylative protonation with wild-type and mutant decarboxylases

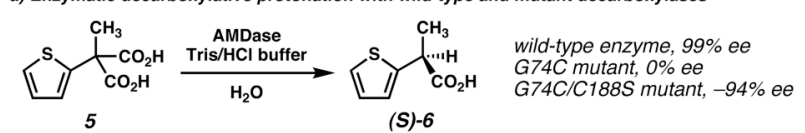

5

(S)-6
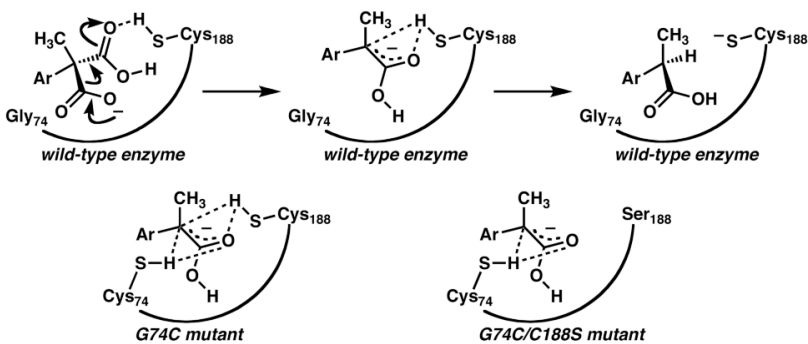

b) Enzymatic oxidation/decarboxylation/protonation/oxidation cascade
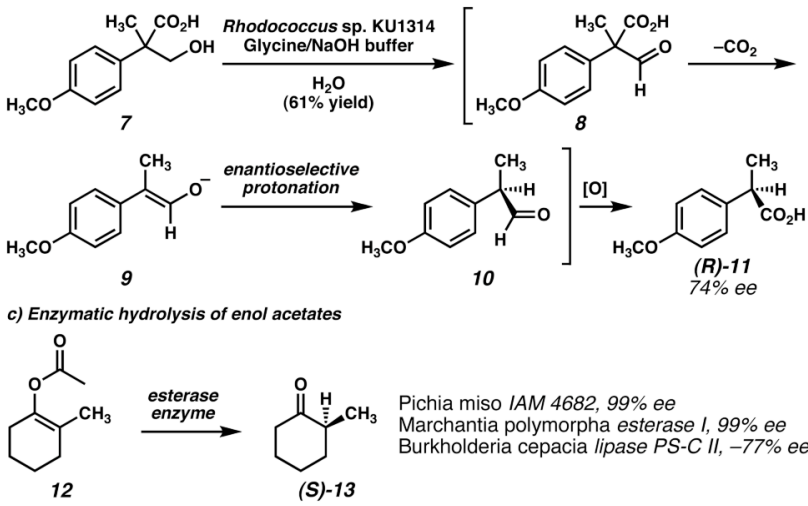

Figure 2. Enzyme-catalyzed enantioselective protonation reactions a) Ohta and coworkers 10, 11 found AMDase transformed arylmalonic acids into enantioenriched arylpropionic acids. Noting the homology of AMDase to racemase enzymes led to the design of mutant enzymes that formed racemic or enantiomeric arylpropionic acids. $15,16 \mathrm{~b}$ ) Ohta and coworkers ${ }^{18}$ identified a Rhodococcus capable of initiating an oxidation/ decarboxylation/protonation/oxidation cascade to convert tropate derivatives to enantioenriched propionic acids. c) Several esterase enzymes have been found to convert enol acetates to enantioenriched ketones.19,20,21 (Reduced to 45\%) 
a) Kim's enolate protonation<smiles>CC1=C(OS(C)(=O)=O)c2ccccc2CC1</smiles>

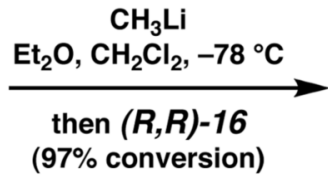

( $97 \%$ conversion)

b) Eames's enolate protonation<smiles>CC(=O)OC1=C(C)CCc2ccccc21</smiles>

17

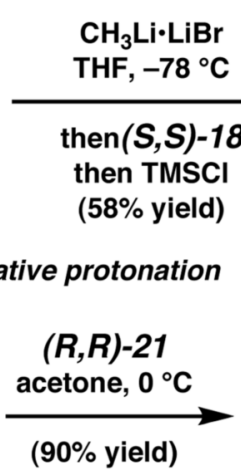<smiles>C#CN1CCCC[C@@H]1C(=O)OCC</smiles>

(S)-20

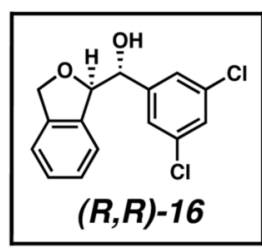
(R)-15 $90 \%$ ee

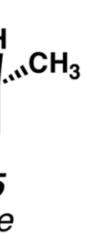

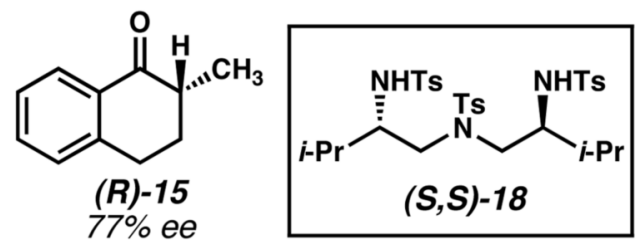

c) Rouden's decarboxylative protonation

19<smiles>CCOC(=O)C1(C(=O)O)CCCCN1C#N</smiles>

9

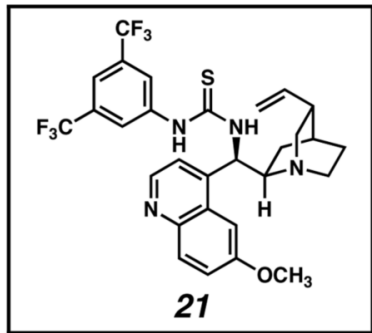

d) Donohoe's partial pyrrole reduction<smiles>COC(=O)n1c(C(C)=O)ccc1C(C)=O</smiles>

22
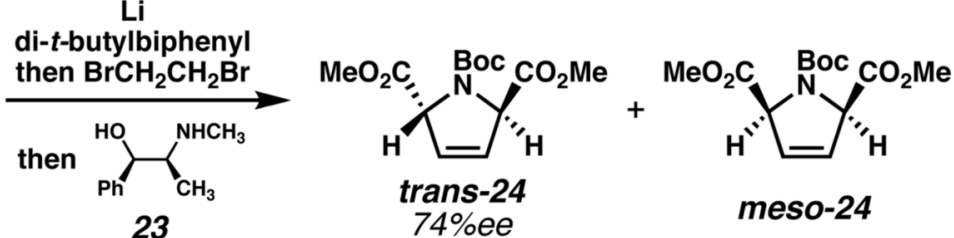

Figure 3. Enantioselective protonations by means of stoichiometric chiral Brønsted acids a) Kim and coworkers ${ }^{23}$ synthesized several chiral alcohols (e.g., 16) to serve as chiral Brønsted acids. High e.e. products could be obtained with tetralone-derived enolates, but lower selectivity was found for substrates lacking an aryl group. b) Eames and coworkers 24,25,26 employed a series of sulfonamide Brønsted acids (e.g., 18) in enantioselective protonation reactions. In some cases an external quench procedure allowed preparation of the antipode of the ketone product. c) Rouden and coworkers ${ }^{27,28}$ found that Cincona alkaloid-derived thioureas (e.g., 21) promoted the decarboxylative protonation of malonate hemiesters. Use of diastereomeric thioureas allowed access to the opposite enantiomer of product with comparable e.e. d) Donohoe and coworkers ${ }^{29,30}$ used amino alcohols (e.g., 23) as proton donors in dissolving metal reductions of pyrroles. In the case of diester 22, a 1:1 mixture of diastereomeric dihydropyrrole products $(\mathbf{2 4})$ was obtained. 

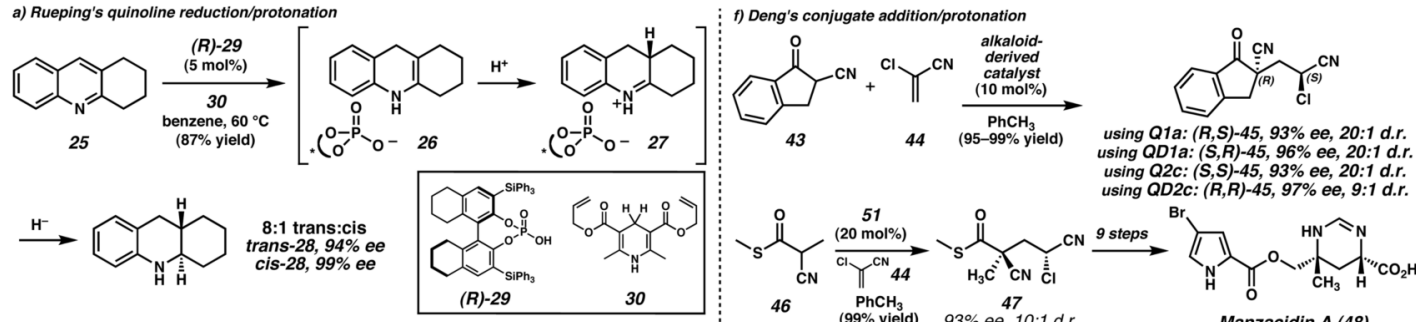
using Q1a: $(R, S)-45,93 \%$ ee, $20: 1$ d.r.
using QD1a: $(S, R)-45,96 \%$ ee, $20: 1$ d.r. using QD1a: $(S, R)-45,96 \%$ ee, $20: 1$ d.r.
using Q2c: $(S, S)-45,93 \%$ ee, 20:1 d.r.
using QD2c: $(R, R)-45,97 \%$ ee, $9: 1$ d.r.

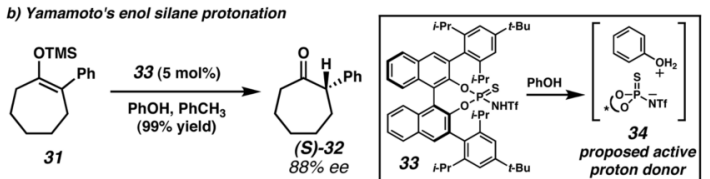

c) Yanagisawa's lithium enolate protonation

OTMS 1. $n$-BuLi, THF, $-78^{\circ} \mathrm{C}$

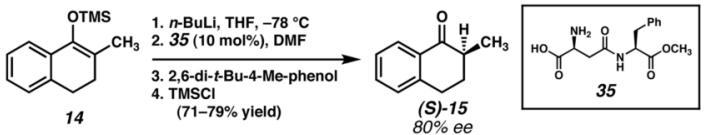

$14 \quad(71-79 \%$ yield $)$

d) Yanagisawa's enol silane protonation
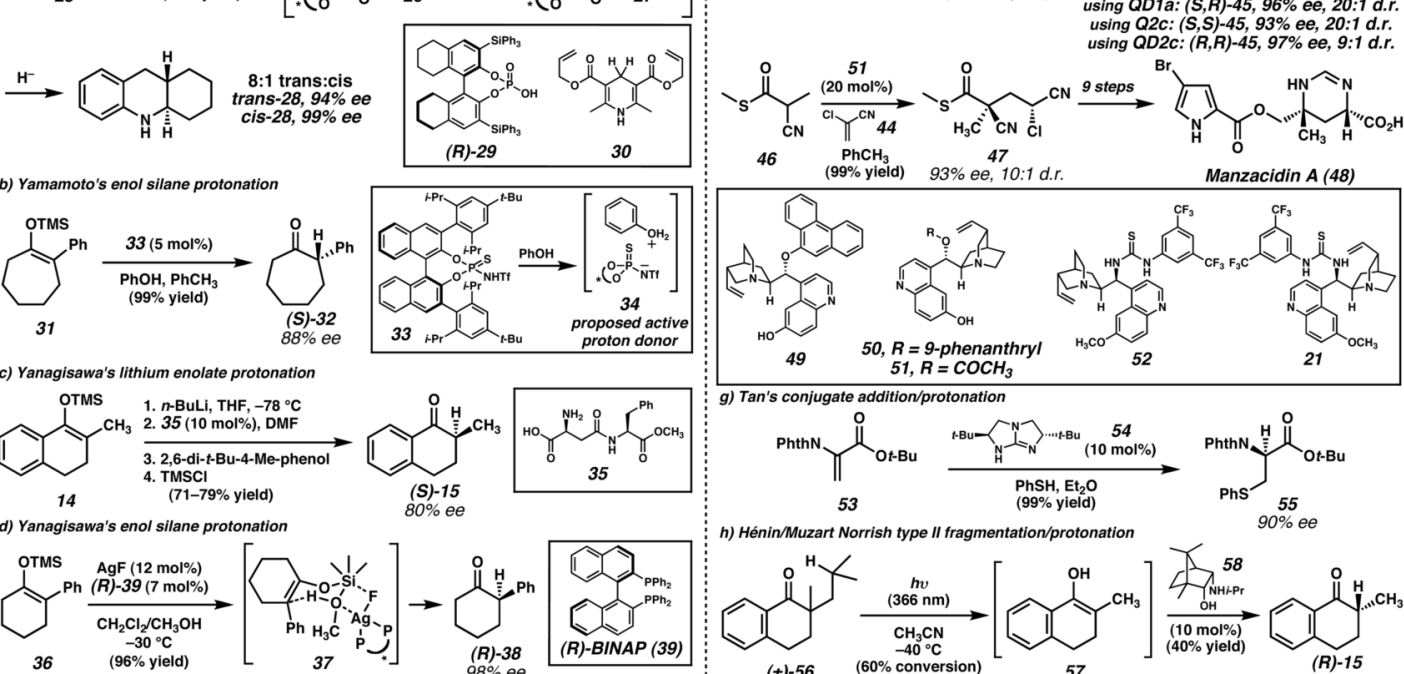

g) Tan's conjugate addition/protonation

PhthN conjugate addition/protent-Bu

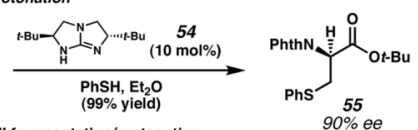

h) Hénin/Muzart Norrish type Il fragmentation/protonation $90 \%$ ee
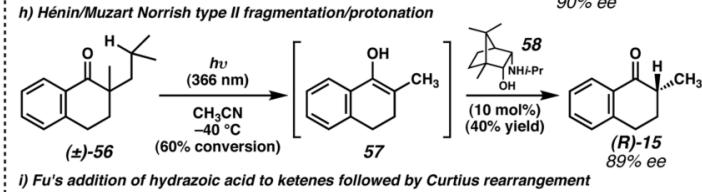

e) Levacher's enol silane protonation

отMS
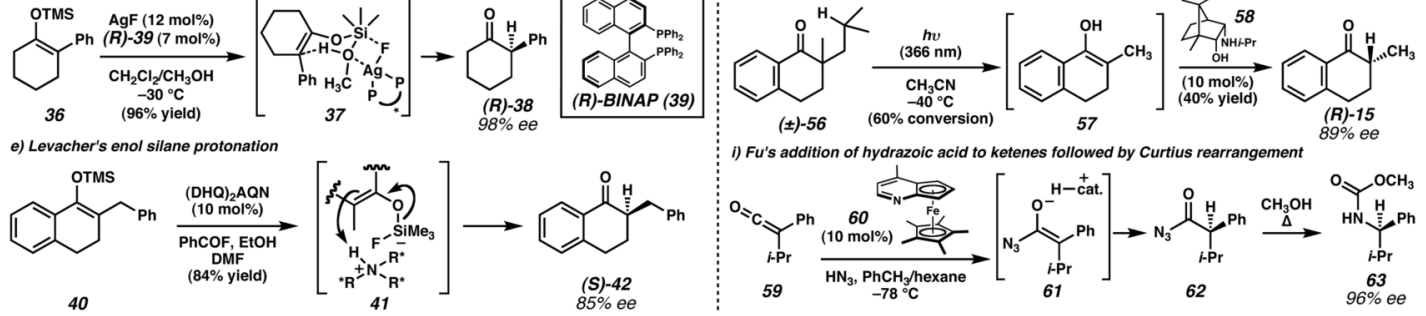

Figure 4. Enantioselective protonations by means of catalytic chiral Brønsted acids

a) Rueping and coworkers ${ }^{31}$ found that chiral phosphoric acid catalysts coupled with Hantzch esters could effect enantioselective enamine protonation in the course of quinoline reductions. b) Cheon and Yamamoto ${ }^{32}$ recently disclosed the use of $N$-triflyl thiophosphoramide catalyst 33 for the conversion of enol silanes to enantioenriched ketones. Empirical observations suggest that the catalyst forms an oxonium ion pair (34) that serves as the active proton donor. c) Yanagisawa and coworkers ${ }^{33}$ used a catalytic amount simple dipeptide $\mathbf{3 5}$ to enantioselectively protonate lithium enolates. Small modifications to the dipeptide caused significant decreases in product e.e. d) A different system developed by Yanagisawa and coworkers 34,35 employed a Ag Lewis acid to activate methanol to serve as a proton donor in the conversion of silyl enol ethers to ketones. An aggregate transition state was proposed for the transformation. e) Levacher and coworkers ${ }^{38}$ reported a protocol for preparing HF in situ. The HF then interacts with a Cinchona alkaloid derived nitrogen base and subsequently activates enol silanes toward protonation by the ammonium species. f) Deng and coworkers ${ }^{39,40}$ demonstrated that catalysts derived from quinine and quinidine were capable of catalyzing addition of prochiral nucleophiles to 2-chloroacrylonitrile (44). All four stereoisomers of the products (e.g., 45) were accessible in high yield, e.e., and diastereomeric ratio by using different catalysts. This transformation was used to carry out an enantioselective formal synthesis of the natural product manzacidin A (48). g) Tan and coworkers ${ }^{42}$ developed enantioselective conjugate addition/protonation sequences with heteroatom nucleophiles in the presence of guanidine catalyst 54. h) Hénin, Muzart, and coworkers ${ }^{43,44}$ found the photochemical fragmentation of racemic $\alpha$-quaternary ketone 56 generated an enol (57) that could undergo enantioselective conversion to $(R)$-2-methyltetralone $((R)-\mathbf{1 5})$ in the presence of amino alcohol catalyst 58. i) Fu and coworkers employed planar-chiral heterocycle catalyst 60 to promote addition of hydrazoic acid to ketenes (e.g., 59). Subseqeunt Curtius rearrangement generated chiral carbamate products (e.g., 63) with high e.e. (Reduced to 45\%) 
a) Fu's Addition of Alcohols to Ketenes

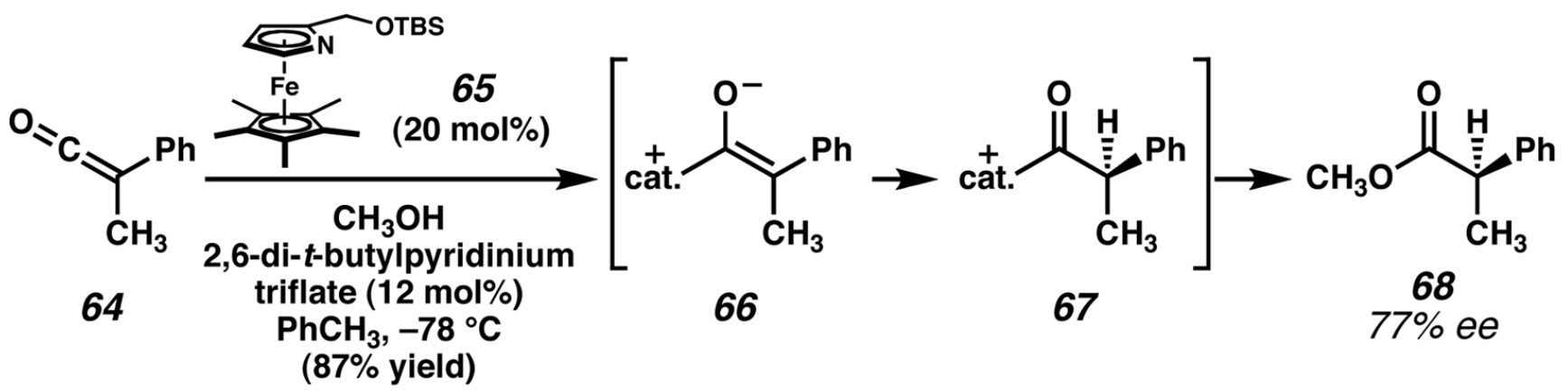

b) Rovis' Protonation of Chloroenolates

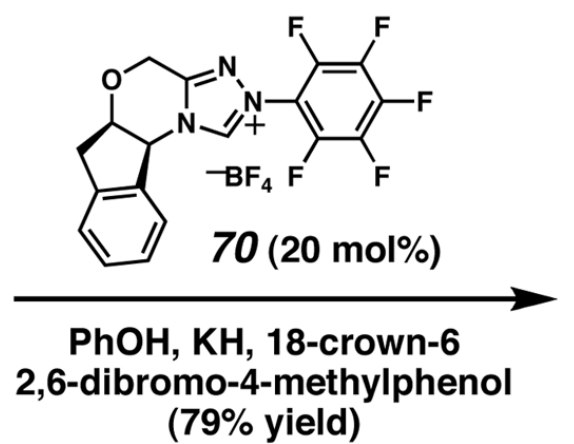<smiles></smiles><smiles>O=CC(Cl)Cc1ccccc1</smiles>

72

$93 \%$ ee

c) Scheidt's Protonation of Homoenolate Equivalents<smiles>C/C(=C\C=O)c1ccccc1</smiles>

73<smiles>Cc1cc(C)c(N2C=[N+](c3ccccc3)[C@H](Cc3ccccc3)C(c3ccccc3)(c3ccccc3)OC2)c(C)c1</smiles>

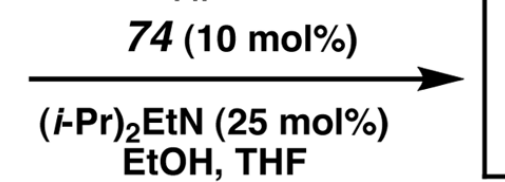

(58\% yield)<smiles>C/C(=C\C(O)=C1/C[C@@H]2C(=NN1C)COC2(c1ccccc1)c1ccccc1)c1ccccc1</smiles>

75<smiles>CCOC(=O)C[C@H](C)c1ccccc1</smiles>

76

$55 \%$ ee

$+$<smiles>CCOC(=O)/C=C(\C)c1ccccc1</smiles>

77

3.4:1 mixture of $76: 77$

Figure 5. The use of nucleophilic heterocycle catalysts to generate chiral proton acceptors

a) $\mathrm{Fu}$ and coworkers ${ }^{50}$ employed azaferrocene catalyst $\mathbf{6 5}$ to achieve enantioselective addition of alcohols to ketenes. Experimental observations suggest that the mechanism differs from the related reaction in Figure 4i. b) Rovis and coworkers ${ }^{53}$ found that triazolium salt $\mathbf{7 0}$ catalyzed the transformation of $\alpha, \alpha$-dichloraldehydes (e.g., 69) into synthetically useful enantioenriched mono-chloroaldehydes (e.g., 72). c) Scheidt and coworkers ${ }^{54}$ achieved enantioselective protonation of homoenolate equivalents with triazolium salt catalyst 74. This demonstrated that enantioselective protonation could occur at a site distant from the chiral catalyst. (Reduced to $45 \%$ ) 

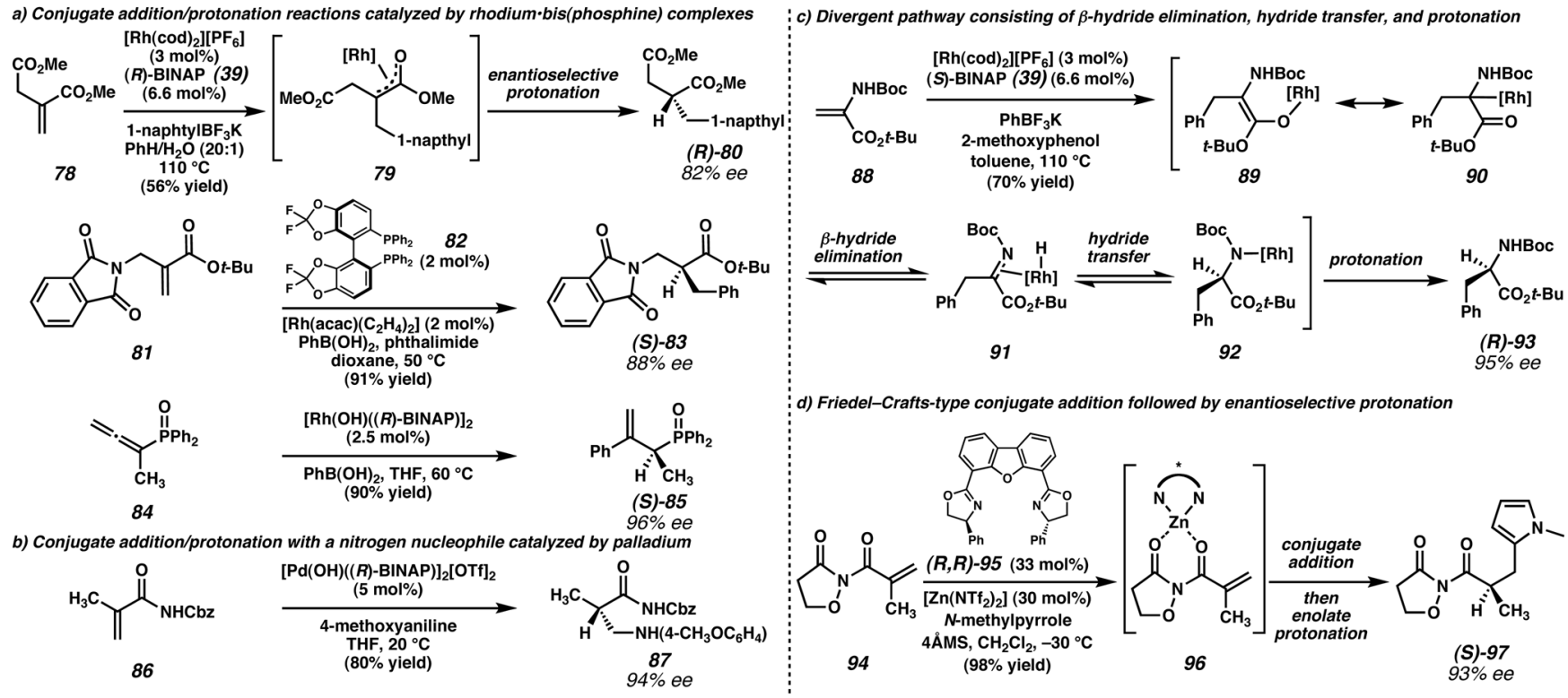

Figure 6. Conjugate addition/protonation sequences catalyzed by chiral metal complexes a) Several other research groups57,55,58,59 have explored Rh-catalyzed conjugate addition/ protonation sequences with various activated electrophiles and carbon-based nucleophiles. b) Sodeoka and coworkers60 found that nitrogen nucleophiles could undergo enantioselective conjugate addition/protonation in the presence of a cationic Pd catalyst. c) Darses, Genet, and coworkers61,62 successfully synthesized $\alpha$-amino acid derivatives through a conjugate addition/protonation sequence, although later mechanistic evidence suggested a different reaction mechanism than they originally postulated. d) Sibi and coworkers ${ }^{63}$ achieved enantioselective protonation of $\mathrm{Zn}$-enolate generated by Friedel-Crafts-type addition of a pyrrole to an acrylimide. (Reduced to $45 \%$ ) 

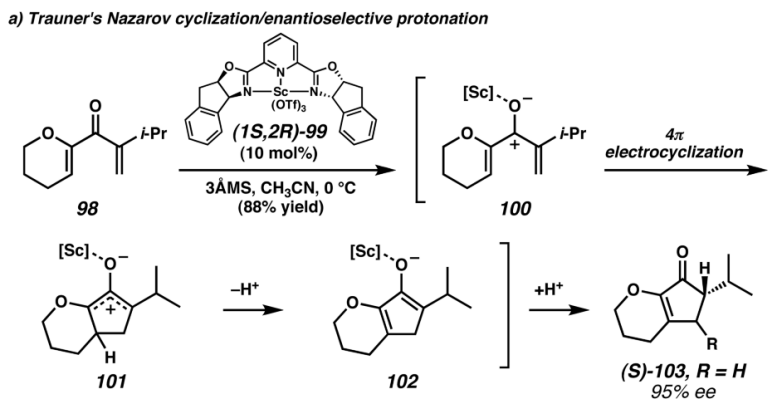

b) Palladium-catalyzed decarboxylative protonation reactions
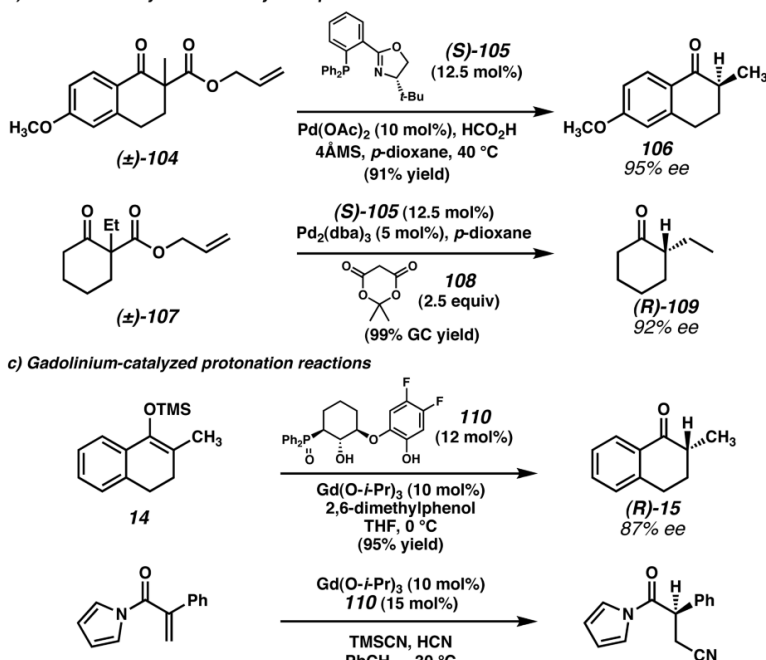

111
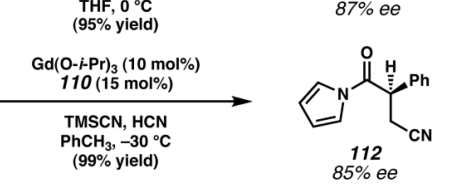

Figure 7. Transition metal-catalyzed enantioselective protonation reactions by means of chiral metal enolates

a) Trauner and coworkers ${ }^{64}$ developed a scandium-catalyzed cascade Nazarov cyclization/ enolate protonation sequence to access cyclopentenones. b) Stoltz and coworkers ${ }^{69,73}$ employed a chiral palladium complex to effect decarboxylative protonation reactions from allyl $\beta$-ketoester substrates (e.g., 104 and 107). Two different proton sources were effective in this transformation: formic acid in the presence of molecular sieves, and Meldrum's acid (108). c) Kanai, Shibasaki, and coworkers ${ }^{75}$ found that a chiral gadolinium complex was useful for enantioselective protonation of enolates generated from either enol silanes or conjugate addition of cyanide to $N$-acryloyl pyrroles. (Reduced to $45 \%$ ) 
<smiles>C=C(CN1C(=O)c2ccccc2C1=O)C(=O)OCC</smiles>

81

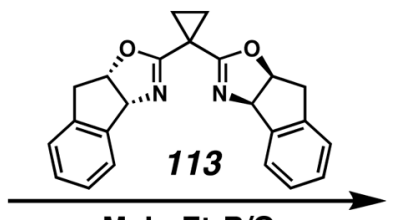

$\mathrm{Mgl}_{2}, \mathrm{Et}_{3} \mathrm{~B} / \mathrm{O}_{2}$ cyclopentyl iodide $\mathrm{CH}_{2} \mathrm{Cl}_{2}-78^{\circ} \mathrm{C}$

(74-86\% yield)<smiles></smiles>

1 equiv $\mathrm{Mgl}_{2} \cdot 113,94 \%$ ee 0.3 equiv $\mathrm{Mgl}_{2} \cdot 113,47 \%$ ee

Figure 8. Radical conjugate addition followed by enantioselective $\mathbf{H}$-atom transfer Sibi and coworkers76,77 demonstrated that Mg•bis(oxazoline) complexes effectively promoted radical conjugate addition to enoates followed by enantioselective quenching of the radical intermediate by $\mathrm{H}$-atom transfer. High e.e. was observed with stoichiometric amounts of Lewis acid, but in some cases substoichiometric amounts of Lewis acid led to significantly lower product e.e. 